\title{
Innovative Strategies for Acquiring IT Applications Among Selected Companies in Metro Manila, Philippines and Its Benefits
}

\author{
Harvey T. Ong \\ De La Salle University-Manila, Metro Manila, Philippines
}

\begin{abstract}
System development life cycle (SDLC) is the systems development and improvement that organizations are used for IT projects. After the company successfully justified an IT investment, it must then decide how to pursue it. Companies have several options for acquiring IT applications, some of their strategies are buying applications, leasing them, using open-source software, using software-as-a-service, developing them in-house, or outsourcing them, and other innovative organization's strategies in acquiring IT applications. The objective of the study is to identify and describe the innovative organization's strategies adopted by selected top corporations in Metro Manila, Philippines in acquiring IT applications. The study likewise aims to look into perceived benefits considered by these companies in their decision to implement the strategies. The research design used was descriptive. Data gathered (survey and interview results) from the respondent companies were used to discuss the innovative strategies in acquiring IT applications and benefits which were perceived by 58 companies in Metro Manila, namely in the following sectors: service; manufacturing; and merchandising corporations. Based on the surveyed and interviewed, the proponent found out that there are 54 company respondents (or 93.10\%) mentioned that they bought the IT applications or off-the-shelf approach. While 26 companies (or 44.83\%) stated that they lease the IT applications. On the other hand, 30 company respondents (or 51.72\%) mentioned they used Open Source, 24 companies (or 41.38\%) used software-as-a-service to acquire their IT applications. There are also 27 companies (or 46.55\%) mentioned that they developed in-house IT applications. All the companies need to apply appropriate organization strategies in acquiring IT applications in able to come up with more successful system development project, in selecting an acquisition strategy, the decision must be made by presenting the feasibility information for each acquisition strategy in which they can be compared easily.
\end{abstract}

Keywords: system development life cycle (SDLC), innovative strategies, IT applications, information technology, IT acquisition

\section{Introduction}

\section{Background of the Study}

During the design phase, the project team also needs to consider three approaches to create the new system,

Harvey T. Ong, Professor, Decision Sciences and Innovation Department, De La Salle University-Manila.

Correspondence concerning this article should be addressed to Harvey T. Ong, 2401 Taft Avenue, Pasay, Metro Manila, Philippines. E-mail: harvey.ong@dlsu.edu.ph. 
which including developing a custom application in-house; buying a packaged system and customizing it; and relying on an external vendor, developer, or system provider to build and/or support the system. Custom development allows developers to be flexible and creative in the way they solve business problems, and it builds technical and creative in the way they solve business problems, and it builds technical and functional knowledge within the organization. Many companies have a development staff that is already over-committed to filling huge backloads of system requests, however, and they just do not have time to devote to a project for which a system is built from scratch. It can be much more efficient to buy programs that have been created, tested, and proven, and a packaged system can be bought and installed in a relatively short period of time, when compared with a custom solution. Workarounds can be used to meet the needs that are not addressed by the packaged application. The design strategy is to outsource the project and pay an external vendor, developer, or service provider to create the system. It can be a good alternative for how to approach the new system, however, it does not come without costs. If a company decides to leave the creation of a new system in the hands of someone else, the organization could compromise confidential or lose control over future development (Dennis, Wixom, \& Roth, 2010).

If a company has successfully justifies an IT investment, it must then decides how to pursue it. Companies have several options for acquiring IT applications (Rainer \& Turban, 2009).

\section{Rationale of the Study}

The proponent's interest in the topic of information system planning began when the proponent embarked on teaching COMP1BU and COMP2BU (Computer Application for Business Management Students), ENTEMIS (Management Information System for Entrepreneurship), SYSTAND (System Analysis and Design), IS Planning, and BUSIMIS (Management Information System for Business Management Students) for undergraduate of the Decision Sciences and Innovation Department, De La Salle University-Manila. And besides, the undergraduate degree of proponent was Computer Science specialized in software technology. The proponent also planned to teach courses like INNOTEC (Innovation in Technology) in the near future. Lastly, the proponent has the interest to look into the innovative organization strategies in acquiring for IT applications among selected companies in Metro Manila, Philippines and its benefits. It is worth knowing because the IT acquisition strategy adopted will determine the success or failure of a System Development Project. In addition, the uses of information system constantly helped companies to improve the way they conduct business transaction and make sure that it continues to meet their company's goals and objectives, and to cut costs and increase profits. Using new computerized system has changed the way that the company works in recent years.

\section{Statement of the Problem}

What are the innovative organization's strategies adopted by the selected top corporations in Metro Manila, Philippines in acquiring information technology applications? And what are the benefits of these strategies as perceived by the companies?

\section{Objectives of the Study}

The objective of the study is to identify and describe the organization's strategies adopted by the selected top corporations in Metro Manila, Philippines, in acquiring for IT applications. The study likewise aims to look into the perceived benefits considered by these companies in their decision to implement the strategies. 


\section{Significance of the Study}

The result of the study will benefit the following:

(1) Faculty of Decision Sciences and Innovation Department and Computer Science-De La Salle University-Manila

This will give the faculty teaching COMP1BU, COMP2BU, ENTEMIS, BUSIMIS, SYSTAND, and INNOTEC an idea on what specific topic needs to include or discuss in class lecture, so the students would be able to apply its application in real business situation when they graduate.

(2) Different corporations in Metro Manila, Philippines

To give them feedback on how different companies in Metro Manila adopt the organization strategies during the acquiring IT applications phase of their system development project. Furthermore, this would also give them an idea on how to further improve in the acquiring of IT applications to make their system development project more successful. Their new created system definitely can help them promote and facilitate their business, streamline their processes and enhance the way conduct their business transaction.

\section{Scope and Limitation}

The company respondents focused on this study will be limited to 58 top corporations in Metro Manila, Philippines.

The data gathering were assisted by proponent' students in entrepreneurship information system class (1st term AY 2010-2011), and it was limited to 100 top corporations based on their gross revenue in Metro Manila, Philippines was stated in Business World Magazines published in early 2010. Business World Top 1,000 Corporations in the Philippines is published annually by Business World Publishing Corporation, with editorial offices at 95 Balete Drive Extension, New Manila, Quezon City, Metro Manila, Philippines.

At first, the limitation of the study was limited to top 100 corporations based on their gross revenue which was stated in business world magazines, but unfortunately, not all the 100 corporations responded. Some of them are not willing to be surveyed nor interviewed. Out of 100, only 58 corporations responded. In addition, only these 58 companies were accessible and located in Metro Manila. And these are composed of 22 service companies, 10 manufacturing companies, and 26 merchandising companies.

The respondent also had a hard time to assess the data gathered from the companies. The data gathered were presented in narrative explanation format, this give the proponent a hard time in coding the data. Another limitation of the study was that there are some perceived benefits of acquiring IT application strategy adopted and other important details and information were not mentioned or discussed clearly by the interviewee respondent of the corporation. And many companies also claimed that this information is kept confidential by their companies.

\section{Framework}

\section{Conceptual Framework}

The conceptual framework of the study is based on (or adopted from) R. Kelly Rainer Jr. and Efraim Turban who stated the following:

Companies have several options for acquiring IT applications, including buying the applications, leasing them, using open-source software, using software-as-a-service, developing them in-house, or outsourcing them (see Figure 1). 


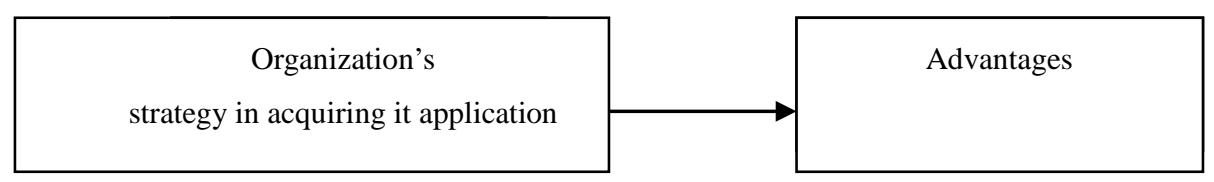

Figure 1. Schematic diagram of the conceptual framework.

Rainer and Turban (2009) stated the following:

(1) Buying the applications-This is also called off-the-shelf approach. The standard features required by IT applications can be found in many commercial software packages. Buying an existing package can be a cost-effective and time-saving strategy compared with developing the application in-house. Nevertheless, the buy option should be carefully considered and planned to ensure that the selected package contains all the features necessary to address the company's current and future needs, otherwise, such packages can quickly become obsolete. You will decide the features that a selected package must have to be suitable.

(2) Lease the application-Leasing can be done in one of three ways: the first way is to lease the application from a software developer and install it on the company's premises. The vendor can help with the installation and frequently will offer to contract for the support and maintenance of the system. Many conventional applications are leased this way; the second way is to use an application service provided (ASP) which becoming more popular; and the third way is to utilize software-as-a-service.

(3) Use open-source software-An organization can obtain a license to use an open-source software product and either use it as is or customize it to develop applications.

(4) Utilize software-as-a-service-Software-as-a-service (SaaS) refers to a method of delivering software in which a vendor hosts the applications. Customers access these applications over a network, typically the Internet, and have no control over the applications. Customers do not own the software, rather, they pay for using it.

(5) Develop the applications in-house-In-house development can make use of various methodologies. The basic—backbone methodology is the system development life cycle (SDLC).

\section{Operational Framework}

Adopted from the Conceptual Framework of Stairs and Reynolds (2009), the organization's strategy adopted by selected top corporations in Metro Manila and its perceived advantages in acquiring IT applications will serve as the variables of this study in terms of:

\section{Operational Definition of Terms}

- Acquisition strategy - The new system will be built and implemented by the project team. There are some ways to approach the creation of a new system: develop a custom application in-house; buy a packaged system and customizing it; and rely on an external vendor, developer or service provider to build the system.

- Information system-A set of interrelated components that collect, manipulate, store, and disseminate data and information and provide a feedback mechanism to meet an objective.

- Outsourcing - general means hiring the services of another organization or individual to perform some of the work that otherwise would be performed by you and your employees.

- Software as a service (SaaS)—An organization that offers the use of software through communication lines is called an application service provider (ASP).

- System development life cycle (SDLC) - Traditional structures framework, used for large IT projects, that consists of sequential processes by which information systems are developed. 


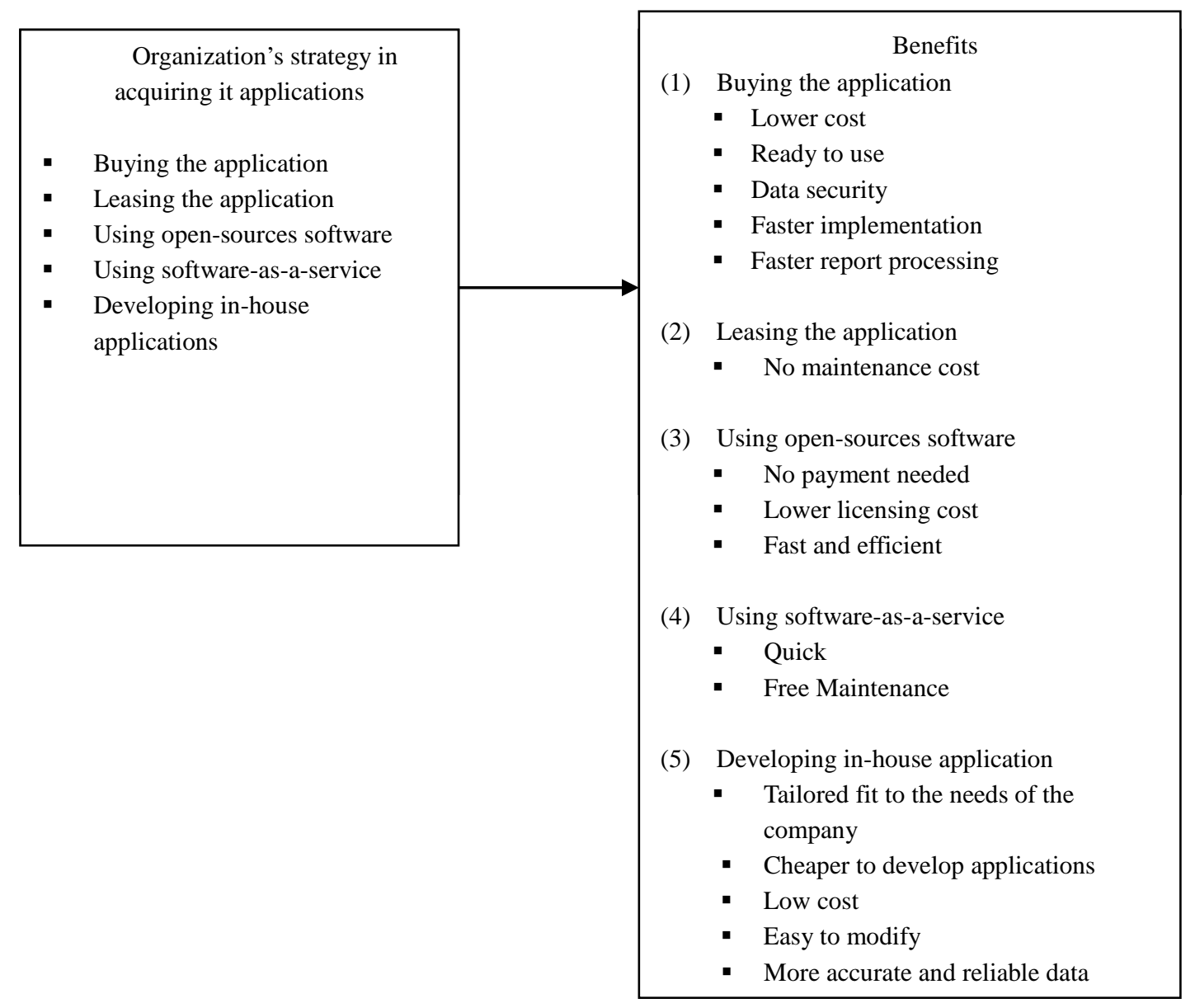

Figure 2. Variables of the study.

\section{Research Methodology}

\section{Research Design}

The research design used was descriptive. The data gathered (survey and interview results) from the respondent companies will used to identify and describe the organization strategies adopted by the selected top corporations in Metro Manila, Philippines in acquiring IT applications. The study will also look into the perceived benefits considered by the 58 corporations in Metro Manila.

Among the 100 list of corporations, only 58 companies responded. Twenty two companies (or 37.93\%) were service companies which includes oil refineries, electric distribution, wireless service, banking, power service, port management, media, financial institution, utility, real estate, telecommunications, transportation, infrastructure, water, call center, and insurance companies. Ten companies (or 17.24\%) responded were manufacturing companies which includes food, automotive, agriculture, beverage and beers, pharmaceutical, pediatric nutrition, cement, packaging companies. And 26 companies (or 44.83\%) were merchandising companies which include shopping and retail, supermarket, warehousing, beauty products, LPG and petroleum companies.

\section{Sampling Plan}

The secondary data were collected during 1st term academic year 2010-2011 from the corporation 
interviewed by ENTEMIS students as the basis of data for this research study.

\section{Method of Data Analysis}

Primary data were tabulated in a data set, and the data were analyzed using the frequency and percentage distribution. Since the data gathered presented in narrative paragraph form, content analysis will be used by the proponent in coding the data. And the data were also presented in frequency distribution table format and context narrative discussion.

\section{Research Findings}

Based on the conceptual framework adapted by the proponents:

The purpose of using the conceptual framework stated in the previous section is to identify and describe the organization strategies adopted by the selected top corporations in Metro Manila, Philippines in acquiring IT applications. The study will also look into the perceived benefits considered by the selected corporations in Metro Manila. This would also describe the social context and corporate culture of the companies studied-the values and beliefs that determine what is admissible and possible within the culture of their corporations involved.

\section{Organization's Strategy Adopted by the Top Corporations in Acquiring IT Applications}

The subjects for the present study are students (including full-time undergraduates, full-time master students, and full-time doctor students) that live in a university in Beijing, and they have received merchandise on-campus from Dangdang and Weilan online bookstores and Amazon and also have on-line shopping experience. The subjects are randomly chosen, and in order to avoid repetition the online shops that are often visited by the subjects were controlled. The present research distributed 300 questionnaires and 230 valid samples are collected with the rate of reclamation of $76.67 \%$, which accord with the requirements of SEM.

Identify applicable sponsor/s here (sponsors).

In Table 1, it shows that 54 respondents out of 58 respondents (or 93.10\%) mentioned that they adopted the "buying the application" strategy in acquiring their IT applications. On the one hand, 26 respondents (or 44.83\%) stated that they used "leasing the application" as their IT applications acquiring strategy. On the other hand, 30 respondents (or 51.72\%) mentioned that they used open sources. There were also 24 companies (or 41.38\%) used the software-as-a-service, and 27 companies (46.55\%) developed in-house applications.

Table 1

Frequency and Percentage Distribution Results With Regards to the Organization's Strategy Adopted by the Top Corporations in Acquiring IT Applications

\begin{tabular}{lll}
\hline & Frequency $(n=58)$ & $\%$ \\
\hline Buying the application & 54 & $93.10 \%$ \\
Leasing the application & 26 & $44.83 \%$ \\
Using open-source & 30 & $51.72 \%$ \\
Using software-as-a-service & 24 & $41.38 \%$ \\
Developing in-house applications & 27 & $46.55 \%$ \\
Others & 10 & $17.24 \%$ \\
\hline
\end{tabular}

Note. Source: Survey and interview conducted with 58 corporations in Metro Manila.

Ten respondents out of 58 respondents (or 17.24\%) mentioned they used other innovative approaches for their conversion strategy, for example, the combination of "developing-in-house applications" and "buying the 
application”.

\section{Perceived Benefits of Adopted Conversion Strategy}

In Table 2, five different company respondents mentioned that their perceived benefits of adopting "buying the application" strategy in acquiring IT application are lower cost, it's ready to use, has data security, faster implementation and roll-out and faster report processing. The company purchases a license to use ready-made application software, they enjoyed the high quality software that is immediately available at low price. It also increased reliability, functionality, and already in use by other firms. They also enjoy that "help" and "training" comes with the software.

Table 2

Frequency and Percentage Distribution Results With Regards to Perceived Benefits of Adopting "Buying the Application" as the Strategy by the Top Companies in Acquiring IT Applications

\begin{tabular}{llc}
\hline & Frequency $(n=54)$ & $\%$ \\
\hline Lower cost & 1 & $1.85 \%$ \\
Ready to use & 1 & $1.85 \%$ \\
Has data security & 1 & $1.85 \%$ \\
Faster implementation and roll-out & 1 & $1.85 \%$ \\
Faster report processing & 1 & $1.85 \%$ \\
\hline
\end{tabular}

Note. Source: Survey and interview conducted with 58 corporations in Metro Manila.

In Table 3, only one company respondent mentioned that their perceived benefits of adopting "leasing the application" strategy in acquiring IT application was there is no maintenance cost. The "lease” option can help company save both time and money. But the leased package may not fit the company's application requirements, however, vendor software often includes the software features that are most commonly needed by the companies in a given industry, the company can decide the features that are necessary. This strategy is attractive to small-medium enterprise (SMEs) that cannot afford large investments in IT.

In Table 4, three different company respondents mentioned that their perceived benefits of adopting "using open source software” strategy in acquiring IT application are no payment needed, lower licensing cost, and fast and efficient. Open source software is software which source code can be easily accessed by the general public.

Table 3

Frequency and Percentage Distribution Results With Regards to Perceived Benefits of Adopting "Leasing the Application" as the Strategy by the Top Companies in Acquiring IT Applications

\begin{tabular}{lll}
\hline & Frequency $(n=26)$ & $\%$ \\
\hline No maintenance cost & 1 & $3.85 \%$ \\
\hline Note. Source: Survey and interview conducted with 58 corporations in Metro Manila. &
\end{tabular}

In Table 5, two different company respondents mentioned that their perceived benefits of adopting "using software-as-a-service” strategy in acquiring IT application are quick and free maintenance. They mentioned that there's no need to allocate hardware for the installation, no need to hire experts for maintenance, and it's available for easy access. An ASP does not install any software on a client's computer, but rather, the application is installed at the ASP's location, along with the databases and files that the application processes for the client. The client needs to pay monthly fees based on the type of application uses, and its employees use 
the applications via a network. The companies do not need to hire, train, or retain a large IT staff.

In Table 6, three different company respondents mentioned that their perceived benefits of adopting "developing in-house applications" strategy in acquiring IT application-tailored fit to the needs of the company. Four other companies mentioned: cheaper to develop applications, low cost, easy to modify, and more accurate and reliable data. Company has a specific response to specialized business needs, in-house IT staff can maintain their software. There's an innovation that may give firm a competitive advantage, and the companies have the pride of ownership.

Table 4

Frequency and Percentage Distribution Results With Regards to Perceived Benefits of Adopting "Using Open Source Software" as the Strategy by the Selected Top Companies in Acquiring IT Applications

\begin{tabular}{lll}
\hline & Frequency $(n=30)$ & $\%$ \\
\hline No payment needed & 1 & $3.33 \%$ \\
Lower licensing cost & 1 & $3.33 \%$ \\
Fast and efficient & 1 & $3.33 \%$ \\
\hline
\end{tabular}

Note. Source: Survey and interview conducted with 58 corporations in Metro Manila.

Table 5

Frequency and Percentage Distribution Results With Regards to Perceived Benefits of Adopting "Using Software-as-a-Service" as the Strategy by the Selected Top Companies in Acquiring IT Applications

\begin{tabular}{lll}
\hline & Frequency $(n=24)$ & $\%$ \\
\hline Quick & 1 & $4.17 \%$ \\
Free maintenance & 1 & $4.17 \%$ \\
\hline
\end{tabular}

Note. Source: Survey and interview conducted with 58 corporations in Metro Manila.

Table 6

Frequency and Percentage Distribution Results With Regards to Perceived Benefits of Adopting "Developing in-house Applications" as the Strategy by the Selected Top Companies in Acquiring IT Applications

\begin{tabular}{llc}
\hline & Frequency $(n=27)$ & $\%$ \\
\hline Tailored fit to the needs of the company & 3 & $11.11 \%$ \\
Cheaper to develop applications & 1 & $3.70 \%$ \\
Low cost & 1 & $3.70 \%$ \\
Easy to modify & 1 & $3.70 \%$ \\
More accurate and reliable data & 1 & $3.70 \%$ \\
\hline
\end{tabular}

Note. Source: Survey and interview conducted with 58 corporations in Metro Manila.

\section{Conclusion/Observation}

The proponent embarked on this study in order to list the organization strategy adopted by the companies in acquiring IT applications based on the surveyed and interviewed from 58 corporations, and to disseminate them in the academic and business community.

Based on the survey and interview, the proponent found out that there are 54 company respondents (or 93.10\%) mentioned that they bought the IT applications or off-the-shelf approach. While 26 companies (or 44.83\%) stated that they just lease the IT applications. On the other hand, 30 company respondents (or 51.72\%) mentioned they used open source, 24 companies (or 41.38\%) used software-as-a-service to acquire their IT applications. There are also 27 companies (or 46.55\%) mentioned that they developed in-house IT applications. There were also perceived benefits which depend on different organization strategies in adapting the acquisition 
for IT applications.

Based on proponent's observation, in these changing times, innovative approaches to IT application acquisition strategies are considered very important in system design stage of software development life cycle. The proponent would like to quote the statement of Dennis, Wixom, and Roth (2010) that:

Each of the acquisition strategies has its strengths and weaknesses, and no one strategy is inherently better than the others (see Table 7). Thus, it is important to consider such issues as the uniqueness of business need for his system, the amount of in-house experience that is available to build the system and the importance of the project skills to the company. Also, the existence of good project management and the amount of time available to develop the application pay a role in the selection process. (p. 264)

Table 7

Comparison of Commonly Used System Acquisition Strategy

\begin{tabular}{|l|l|l|l|}
\hline & Buying the application & Outsourcing & Developing in-house applications \\
\hline Time frame & Short; immediate use & Short; flexible & Flexible; might take a span of time \\
\hline Cost & Usually expensive & Not that cheap or expensive & Cheaper \\
\hline Business needs & Common & Not core to the business & Unique; customize \\
\hline Project management & $\begin{array}{l}\text { Project has a project manager who } \\
\text { can coordinate vendor's efforts }\end{array}$ & $\begin{array}{l}\text { Project has a project manager who woject has a project manager and } \\
\text { will do the outsourcing deal }\end{array}$ & $\begin{array}{l}\text { Project } \\
\text { a proven project methodology }\end{array}$ \\
\hline
\end{tabular}

Nowadays, companies are becoming competitive. Through this, all the companies need to apply appropriate organization strategies in acquiring IT applications in able to come up with more successful system development project, in selecting an acquisition strategy, the decision must be made by presenting the feasibility information for each acquisition strategy in which they can be compared easily. The project team must be able to request for proposal, information and quotations before making acquisition decision, these are also ways to gather reliable information regarding different acquisition strategies alternatives. Proper acquisition strategies will reduce the unnecessary cost and prepare the people in organizations to meet challenges and to respond to the dynamics of the work environment with technological skills and a high level of thinking skills, and to achieve their corporate goals and objectives.

\section{References}

Alter, S. (1999). Information systems: A management perspective 3/e. Benjamin/Cummings Publishing Company. Dennis, A., Wixom, B., \& Roth, R. (2010). System analysis and design. N.J.: John Wiley and Sons, Inc..

Haag, S. (2001). MIS for the information age (2nd ed.). McGraw Hill.

Kendall, K., \& Kendall, J. (2005). System Analysis and Design (Sixth Edition). Paerson Education, Inc..

Koenke, D. (2010). MIS essential. Prentice Hall.

Laudon, K., \& Laudon, J. (1998). Management information systems: New approaches to organization and technology (5th ed.). USA: Prentice-Hall, Inc.

Meleod, R. (1999). Management information system (7th ed.). USA: Prentice-Hall, Inc..

O’Brien, J. (2007). Introduction to information systems, 13/e. McGraw-Hill Higher Education.

Rainer, R. K. Jr., \& Turban, E. (2009). Introduction to information system: Enabling and transforming business. N.J.: John Wiley and Sons, Inc..

Stair, R., \& Reynolds, G. (2009). Introduction to information system. USA: Course Technology Press.

Turban, E., et al. (2001). Introduction to information technology. N.J.: John Wiley and Sons, Inc.. 